\title{
De la théorie de l'émission dans une vente à distance par correspondance classique à la théorie de la réception dans une vente à distance électronique
}

\author{
Hicham Rahal, \\ Enseignant-Chercheur à École Nationale de Commerce et de Gestion \\ Université Ibn Tofail, Kenitra, Morocco
}

Doi:10.19044/esj.2020.v16n19p433 URL:http://dx.doi.org/10.19044/esj.2020.v16n19p433

\section{Résumé}

La vente à distance entre absents est un concept ancien qui mute face aux nouvelles technologies de l'information. On se demande, si réellement il y a une évolution, en droit marocain,du régime juridique appliqué dans une vente à distance ( VAD) entre absents (vente par correspondance classique et vente électronique utilisant le système de double clic). La méthodologie utilisée s'est basée sur une confrontation entre les deux régimes juridiques celui du dahir des obligations et contrats et ceux des lois : loi n ${ }^{\circ} 31-08$ relative à la protection du consommateur et loi $\mathrm{n}^{\circ} 53-05$ relative à l'échange électronique de données juridiques, avec une analyse critique dudit cadre juridique marocain. Il ressort de l'étude que le moment de formation du contrat dans une VAD diffère que l'on est face à une VAD par correspondance par lettre classique qui est encadrée par les articles 23 à 32 du DOC de 1913, soit que l'on se trouve face à une VAD dématérialisée utilisant notamment l'outil internet et le système de double clic. Les résultats dégagés font que dans une VAD par correspondance la doctrine marocaine est unanime à défendre la théorie de l'émission, alors que pour une vente à distance électronique utilisant le système de double clic et sur la base de l'analyse des différentes dispositions juridiques qui encadrent la VAD utilisant des supports électroniques, les dispositions vont dans le sens d'adopter la théorie de la réception.

Mots-clés: Vente à distance dématérialisée, vente par correspondance, évolution doctrinale, théorie de l'émission, théorie de la réception 


\title{
The Theory of Issuance in a Classic Mail Order Distance Selling to the Theory of Reception in an Electronic Distance Selling
}

\author{
Hicham Rahal, \\ Teacher-Researcher at the National School of Business and Management \\ Ibn Tofail University, Kenitra, Morocco
}

\begin{abstract}
Distance selling among absentees is an ancient concept that is rapidly changing in the face of new information technologies. Questions arise around the doctrinal evolution of Moroccan law in regard to the legal regime applied to distance selling (DS) between absentees (selling through classic mail order and e-sales using the double click system). The methodology used was based on a comparison between the two legal frameworks in place and notably the Dahir which refers to obligations and contracts (DOC) and the laws: Law $\mathrm{n}^{\circ}$ 31-08 relating to consumer protection and Law $\mathrm{n}^{\circ}$ 53-05 relating to the electronic exchange of legal data. This include a critical analysis of the Moroccan legal framework aforementioned. In this study, the moment of the formation of a DS contract differs depending on weather it concerns a classic DS mail order, which is governed by articles 23 to 32 of the DOC of 1913, or a dematerialized DS using the Internet tool and the double-click system. The results show that, in a mail order distance selling, the Moroccan doctrine is unanimous in defending the theory of emission. Nevertheless, for an electronic distance selling using the double-click system and on the basis of the analysis of the various legal framework governing distance selling using electronic media, the provisions lean towards adopting the theory of reception.
\end{abstract}

Keywords: Dematerialized distance selling, classical mail order, doctrinal evolution, the theory of emission, the theory of reception

\section{Introduction}

La vente à distance est un contrat, dont les intervenants sont éloignés les uns des autres. Parmi les premières techniques de vente à distance, nous trouvons la vente par correspondance (Shandi, 2005). Le Dahir des Obligations et contrats de 1913 (DOC) est le cadre légal, au Maroc, qui encadre les rapports contractuels entre absents. Ce cadre a subi des amendements pour prendre en compte les nouveaux aspects de la vente à 
distance par le biais d'instruments électroniques, ce qui a eu pour effet l'intégration des dispositions de la loi $\mathrm{n}^{\circ}$ 53-05 relative à l'échange électronique de données juridiques au DOC.

Le concept de vente à distance, tel qu'il se développe actuellement, utilise des données qui transitent d'un point à un autre en transmettant un flux d'informations sur les personnes qui utilisent ces moyens de communication pour contracter, vendre ou acheter. Ces éléments sont des données personnelles de tout individu. Cela met en jeu, entre autres, la sécurité des données personnelles des contractants utilisant les réseaux électroniques. Pour être en phase avec le développement de ces ventes à distances et dans un souci de sécurité de données personnelles et de la vie privée des citoyens, le législateur marocain a édicté la loi $\mathrm{n}^{\circ}$ 09-08 relative à la protection des personnes physiques à l'égard du traitement des données à caractère personnel, promulguée par le Dahir n ${ }^{\circ}$ 1-09-15 du 22 safar 1430 (18 février 2009), et publié au BO, $\mathrm{N}^{\circ}$ 5714-4 du 7 rabii I 1430 (5 mars 2009).

La vente à distance est un système de distribution spécifique dans lequel l'offre de produits ou de services à la clientèle (consommateurs ou professionnels) s'effectue par l'intermédiaire d'un support écrit ou audiovisuel (catalogue, publipostage, Minitel, téléviseur, Internet...). La première dénomination de ce canal «vente par correspondance » a été remplacée par celle de «vente à distance » étant donné la diversité des modes de vente qui ne se font plus uniquement par correspondance au moyen d'imprimés adressés aux clients ou distribués sans adresses dans les boîtes aux lettres (Djlassi, 2000).

Le mot « distance » exprime « la diffusion d'un message commercial à un destinataire éloigné et la non-présence simultanée du vendeur et de l'acheteur au moment de la transaction » (Djlassi, 2000).

La vente à distance, concept ancien, considéré comme une variété de contrats entre absents. KÛHN a donné une définition assez exacte en 1877 : "Abwesenheit im weiteren= oder im rechtsinn susammen-fassen (de l'absence entendue au sens vulgaire et au sens juridique de ce mot), dans jahrbucher fur die dogmatik des heutigen romiseschen und deutchen pruvatrechts», p.8; il souligne que «le contrat entre présents étant celui qui se forme entre des parties qui se trouvent juridiquement au même lieu, il faudra, à l'inverse, définir le contrat entre absents celui qui se forme entre des parties juridiquement éloignées l'une de l'autre » (Valery, 1895).

\section{Méthodologie :}

La méthodologie utilisée pour traiter cet article s'est basée sur une analyse comparative et critique du cadre juridique marocain en matière de vente à distance avant et après la promulgation des lois : sur la protection du consommateur à savoir la loi $\mathrm{n}^{\circ}$ 31-08 et la loi $\mathrm{n}^{\circ}$ 53-05 relative à l'échange 
électronique de données juridiques qui ont été introduites suite à l'ingérence de l'outil électronique notamment internet dans les rapports contractuels. Cet état de fait a eu un impact sur l'application des règles de droit marocaine suivant le cas de figure où le consommateur est confronté à une vente par correspondance ou une vente électronique selon le système de double clic. C'est une confrontation entre deux théories en matière de vente à distance. Aussi, le régime juridique appliqué aura des conséquences différentes selon qu'on soit face à une vente par correspondance ou dans une vente dématérialisée. Pour se faire l'article s'est focalisé sur les points suivants : 1) le cadre général, 2) le regard de la doctrine marocaine sur la vente par correspondance classique et 3) l'évolution doctrinale quant à la théorie appliquée avec l'introduction de la vente à distance dématérialisée, électronique, utilisant le système de double clic en droit marocain et l'analyse critique faite sur cet aspect.

\section{Le cadre général de l'étude}

Dans cet article, il a été question, d'étudier l'évolution doctrinale que connaît le droit civil marocain en matière d'échange du consentement dans une VAD entre absents, le droit marocain est passé d'une théorie à une autre en fonction des outils utilisés dans une VAD entre absents utilisant la correspondance classique et celles utilisant la VAD électronique se basant sur le système d'acceptation de double-clic.

La rencontre des volontés dans les contrats par correspondance classique (lettre) selon le DOC marocain et cela comparativement à l'évolution perceptible dans la théorie prise en considération dans les contrats électroniques à distance avec la loi 31-08, montre une évolution de la théorie utilisée par le passé a savoir celle de l'émission et celle qui domine actuellement dans les ventes dématérialisées, la théorie de la réception.

En effet, plusieurs solutions sont concevables permettant la localisation du contrat par correspondance classique et delà expliciter la rencontre des volontés dans le contrat à distance par correspondance. Le développement qui suit permettra de montrer les différents choix posés en matière de théories doctrinales au droit marocain et la solution qui a été adoptée ou qui se rapproche du cas marocain.

\section{Le regard de la doctrine marocaine sur la vente par correspondance classique}

La doctrine marocaine pose deux hypothèses à savoir (Azziman, 1995):

- On peut d'abord considérer que le contrat se forme lorsque l'acceptant exprime son accord et là où l'acceptant exprime son accord. On se trouve dans deux repères possibles : 
- on peut retenir le moment et le lieu où l'acceptant exprime son accord, c.a.d, le moment est le lieu où la lettre d'acceptation est rédigée. C'est le système de la déclaration ${ }^{29}$ qui présente des inconvénients : il est difficile de prouver à quel moment la lettre a été rédigée et on ne peut empêcher l'auteur de la lettre d'antidater ou de postdater son acceptation. Le système de la déclaration ouvre donc la porte de la fraude.

- on peut considérer que le contrat ne se forme qu'au moment où l'acceptant se dessaisit de sa réponse et là où il se dessaisit de sa lettre. Ce qui revient, pratiquement à retenir le moment où la lettre d'acceptation est expédiée et le lieu où elle est expédiée c'est le système de l'émission ${ }^{30}$. Les problèmes de preuves et de fraudes cessent alors de se poser puisque le tampon de la poste servira à localiser le contrat dans le temps et dans l'espace sans que l'auteur puisse modifier quoi que ce soit.

- On peut aussi estimer que la formation du contrat doit être retardée jusqu'à ce que la lettre d'acceptation soit parvenue à l'auteur de l'offre. Ici on se trouve devant deux possibilités savoir :

- selon le système de l'information ${ }^{31}$, la rencontre des volontés s'opère lorsque l'auteur de l'offre prend connaissance de la lettre d'acceptation. La solution est difficile à admettre, car rien n'empêcherait l'auteur de l'offre de retarder à l'infinie la formation du contrat. De plus, il sera souvent difficile de savoir où et quand la lettre d'acceptation a été réellement ouverte.

- selon le système de la réception, il suffit que l'offrant soit en mesure de prendre connaissance de la lettre d'acceptation. Le moment et le lieu de la formation du contrat seront donc déterminés par la remise de la lettre, mais la remise de la lettre peut poser des problèmes de preuve.

En somme, si l'on écarte les solutions qui soulèvent le plus de difficultés les systèmes de la déclaration et de l'information, le problème revient à choisir entre le système de l'émission et celui de la réception.

Les enjeux qui ressortent du choix entre les différents systèmes se résument ainsi (Azziman, 1995):

- si l'on retient le système de la réception ${ }^{32}$, le contrat se formera au moment et au lieu où la lettre d'acceptation sera remise à l'offrant,

\footnotetext{
${ }^{29}$ Voir aussi pour cet aspect : Jules VALERY 1885 et Maurice AUBERT 1893.

${ }^{30}$ Idem

${ }^{31}$ Ibidem

${ }^{32}$ Ibidem
} 
l'acceptant pourra revenir sur sa décision et annuler la lettre par télégramme ou télex. De plus, si la loi qui régit le contrat vient à être modifiée entre l'émission et la réception. Le contrat sera régi par la loi nouvelle puisqu'il sera formé sous l'empire de la loi nouvelle. Enfin, s'il s'agit d'une vente, les risques resteront à la charge du vendeur (qui demeure propriétaire) jusqu'à la remise de la lettre à l'offrant (formation du contrat et transfert de la propriété) (Azziman, 1995).

- pour le système de l'émission, le contrat se formera au moment et au lieu de l'expédition de lettre. En conséquence, l'acceptant ne pourra plus revenir sur sa décision dès qu'il aura expédié sa lettre. De plus, en cas de modification de la loi postérieurement à l'expédition de la lettre, le contrat sera régi par la loi ancienne puisqu'il aura été formé sous l'empire de la loi ancienne. Enfin si la chose vendue arrive à périr après l'expédition, les risques seront à la charge de l'acquéreur devenu propriétaire (Azziman, 1995).

De manière générale, le choix de l'un ou de l'autre système dépend le moment et le lieu de réalisation de l'accord. Ce moment est important non pas parce qu'il marque le point de départ des obligations issues du contrat, mais aussi parce que c'est à ce moment qu'il faudra se placer pour déterminer la loi applicable (en cas de modification de loi) ainsi que pour apprécier la capacité des parties (Azziman, 1995).

Le droit marocain, le DOC, au niveau de l'article 24 dispose que « le contrat par correspondance est parfait au moment et dans le lieu où celui qui a reçu l'offre répond en l'acceptant.. » et les dispositions de cet article ont toujours été comprises comme consacrant le système de l'émission. On en déduit qu'en droit marocain, le contrat par correspondance se forme au moment où la lettre d'acceptation est expédiée et là où elle est expédiée (Azziman, 1995).

La même règle est d'ailleurs adoptée et appliquée par l'article $24 \mathrm{du}$ DOC au contrat conclu au moyen d'un intermédiaire, messager, mandataire, courtier qui se forme au moment et dans le lieu ou celui qui a reçu l'offre répond à l'intermédiaire qu'il accepte (Azziman, 1995).

Toujours selon la doctrine marocaine, il est souvent difficile, voire impossible, de distinguer le moment de cette acceptation de celui de la conclusion du contrat puisque selon la théorie dominante en droit marocain concernant le critère de localisation, le moment de l'acceptation est, celui même, celui de la formation du contrat (Toumlilt, 2008).

Un constat se dégage, le droit marocain face à la VAD dématérialisée évolue. C'est ce qui a été développé dans le point qui suit : 


\section{L'évolution doctrinale quant à la théorie appliquée avec l'introduction de la vente à distance dématérialisée}

Les régimes juridiques à appliquer seront différents que l'on soit face à une $\mathrm{VAD}$ dématérialisée ou à une $\mathrm{VAD}$ par correspondance (lettre classique) (Rahal, 2018).

Dans une VAD électronique, utilisant le système de double clic, c'est bien le régime du droit de consommation qui aura application; de plus on constate une évolution de la doctrine quant à la théorie qui domine dans ce nouveau concept de VAD dématérialisée, c'est bien la théorie de la réception alors que dans la VAD par correspondance la théorie dominante était celle de l'émission. Alors, que dans une VAD par correspondance classique ce sont les dispositions du DOC de 1913 qui s'appliqueront (Rahal, 2018).

Mais de quelle correspondance s'agit-il? Bien évidemment la correspondance par une simple lettre, car une vente à distance par correspondance électronique ce n'est pas le régime juridique du droit de consommation qui lui sera appliqué, mais bien celui des dispositions introduites à savoir l'article 2-65 de la loi $\mathrm{n}^{\circ}$ 53-05 relative des échanges électroniques des données juridiques, qui sont venues compléter les dispositions du dahir formant code des obligations et contrats (Rahal, 2018).

Ce qui conforte encore plus l'évolution doctrinale quant à la théorie appliquée dans une vente entre absents utilisant les moyens électroniques et la vente par correspondance classique, ce sont bien les dispositions de l'article 65-5, de la loi n ${ }^{\circ}$ 53-05 relative à l'échange électronique de données juridiques ${ }^{33}$, l'alinéa 3 , dudit article stipule que «Le destinataire est irrévocablement lié à l'offre dès sa réception». Cela démontre le couronnement de la théorie de la réception sur celle de l'émission. Le constat qui se dégage va dans le sens d'un glissement du droit marocain vers l'adoption par la doctrine de la domination de la théorie de la réception sur celle de l'émission (Rahal, 2018).

\section{Conclusion}

L'article a exploré l'évolution doctrinale en matière de vente à distance. Deux théories se confrontent, la théorie de l'émission dans une vente

\footnotetext{
${ }^{33}$ Article 65-5. - Pour que le contrat soit valablement conclu, le destinataire de l'offre doit avoir eu la possibilité de vérifier le détail de son ordre et son prix total et de corriger d'éventuelles erreurs, et ce avant de confirmer ledit ordre pour exprimer son acceptation. L'auteur de l'offre doit accuser réception, sans délai injustifié et par voie électronique, de l'acceptation de l'offre qui lui a été adressée.

Le destinataire est irrévocablement lié à l'offre dès sa réception.

L'acceptation de l'offre, sa confirmation et l'accusé de réception sont réputés reçus lorsque les parties auxquelles ils sont adressés peuvent y avoir accès.

Dahir $\mathrm{n}^{\circ}$ 1-07-129 du 19 kaada 1428 (30 novembre 2007) portant promulgation de la loi $\mathrm{n}^{\circ}$ 53-05 relative à 1 'échange électronique de données juridiques
} 
par correspondance classique et la théorie de la réception qui est venue se substituer à l'ancienne théorie pour la vente à distance électronique dématérialisée.

Il ressort de l'étude que le moment de formation du contrat dans une VAD diffère que l'on est face à une VAD par correspondance par lettre classique qui est encadrée par les articles 23 à 32 du DOC de 1913, soit que l'on se trouve face à une VAD dématérialisée utilisant notamment l'outil internet et le système de double clic. Ce qui conforte l'idée de cette évolution juridique c'est bien les différentes théories qu'adopte l'une ou l'autre face à une VAD entre absents.

Pour la VAD dématérialisée, il est plausible de penser qu'il s'agit de la théorie de la réception et cela sur la base d'informations et d'analyses dégagées à travers la lecture de différentes dispositions juridiques qui encadre la VAD utilisant des supports électroniques. Par contre, dans une VAD par correspondance, la doctrine est unanime dans ce sens à défendre une certaine théorie, le moment de formation du contrat est dominé par la théorie de l'émission. On peut déduire qu'il y a bien évidemment une évolution, doctrinale, dans le droit commun marocain en matière du concours de volonté quant à la théorie applicable (Rahal, 2018).

Pour ce qui est des perspectives, il serait judicieux de faire une recherche sur l'effectivité, des règles juridiques introduites en droit marocain, pour la protection du consommateur face à la vente à distance dématérialisée.

\section{References:}

1. Aubert, M. (1893). Du contrat par correspondance, thèse, faculté de droit de Paris, 232 pages.

2. Azziman, O. (1995). Le contrat, droit civil droit des obligations et contrats, Édition Lefennec, 310 pages.

3. Dahir $\mathrm{n}^{\circ}$ 1-02-309 du 25 rejeb 1423(3 octobre 2002) portant promulgation de la loi $\mathrm{n}^{\circ}$ 44-00 complétant du code des obligations et contrats; Bulletin Officiel $\mathrm{n}^{\circ} 5054$ du 2 ramadan 1423 (7 novembre 2002), p.1223.

4. Dahir $\mathrm{n}^{\circ} 1-07-129$ du 19 kaada 1428 (30 novembre 2007) portant promulgation de la loi $\mathrm{n}^{\circ}$ 53-05 relatif aux échanges de données juridiques; Bulletin Officiel n ${ }^{\circ} 5584$ du 25 kaada 1428 (6 décembre 2007), p.1357.

5. Dahir $\mathrm{n}^{\circ} 1-09-15$ du 22 safar 1430 (18 février 2009) portant promulgation de la loi $\mathrm{n}^{\circ} 09-08$ relative à la protection des personnes physiques à l'égard du traitement des données à caractère personnel, BO N $\mathrm{N}^{\circ}$ 5714-4 du 7 rabii I 1430 (5 mars 2009).

6. Dahir $\mathrm{n}^{\circ}$ 1-11-140 du 16 ramadan 1432 (17 août 2011) portant promulgation de la loi $\mathrm{n}^{\circ}$ 24-09 relative à la sécurité des produits et des 
services et complétant le dahir du 9 ramadan 1331 (12 août 1913) formant code des obligations et contrats; Bulletin Officiel $\mathrm{n}^{\circ} 5984 \mathrm{du}$ 8 kaada 1432 (6 octobre 2011), p.2166.

7. Dahir n¹-16-05 du 23 rabii II 1437 (3 février 2016) portant promulgation de la loi ${ }^{\circ}$ 107-12 modifiant et complétant la loi $\mathrm{n}^{\circ} 44-$ 00 relative à la vente d'immeuble en l'état futur d'achèvement; Bulletin Officiel $\mathrm{n}^{\circ} 6518$ du 17 safar 1438 (17 novembre 2016), p.1717.

8. Dahir $\mathrm{n}^{\circ} 1-95-153$ du 13 rabii I 1416 (11 août 1995) portant promulgation de la loi $\mathrm{n}^{\circ} 25-95$ abrogeant l'article $726 \mathrm{du}$ code des obligations et contrats; Bulletin Officiel $n^{\circ} 4323$ du 10 rabii II 1416 (6 septembre 1995), p.602.

9. Dahir $\mathrm{n}^{\circ} 1-95-157$ du 13 rabii I 1416 (11 août 1995) portant promulgation de la loi $\mathrm{n}^{\circ}$ 27-95 complétant le code des obligations et contrats ; Bulletin Officiel $\mathrm{n}^{\circ} 4323$ du 10 rabii II 1416 (6 septembre 1995), p.602.

10. Djlassi S. (2000). «l'influence de la perception temporelle sur le comportement d'achat par correspondance et à distance : application à l'achat par catalogue », "art.», I.A.E. de Dijon disponible sur : http://institut-gestion.univ-larochelle.fr/IMG/pdf/01_djlassi.pdf. Consulté en 2018.

11. Loi $\mathrm{n}^{\circ}$ 31-08 édictant des mesures de protection du consommateur promulguée par le dahir $\mathrm{n}^{\circ} 1-11-03$ du 14 rabii I, 1432. B.O n ${ }^{\circ} 5932 \mathrm{du}$ 7 avril 2011.

12. Rahal, H. (2018). La vente à distance dématérialisée par internet : cas du contrat d'assurance, Thèse de doctorat, spécialité droit des assurances, Université Abdelmalak saidi, Tanger, Maroc, 460 pages.

13. Shandi, Y. (2005). La formation du contrat à distance par voie électronique, thèse, Université Robert Schuman Strasbourg III, 387 pages.

14. Toumlilt Mohammed Diyaâ (2008). Le commerce électronique au Maroc, Aspects juridiques, éditions Maghrébines 2008, 511 pages.

15. Valery, J. (1895). Des contrats par correspondance, du rôle de la correspondance dans l'exécution des contrats, THORIN et fils, éditeurs, Paris, 501 pages. 